\title{
DETECTION OF HUMAN FACES IN COLOR IMAGES AND PERFORMANCE ANALYSIS OF DIFFERENT SKIN CHROMINANCE SPACES AND SKIN CHROMINANCE MODELS
}

\author{
Poorvi Bhatt \\ Health Science Department, Leidos (Legacy Lockheed Martin Information Systems and Global Solutions), USA
}

\begin{abstract}
The overall objective of the project is to build a system that detects human faces in a given color image and to compare performance of different chrominance models and chrominance spaces. For face detection, approach is to detect skin color and segment given image into skin and non-skin regions. In a skin segmented image, the region of skin whose height to width ratio falls under well-known Golden ratio $=(1+\sqrt{5})) / 2 \pm$ some tolerance, the probability of that region to be considered as face is very high. In this implementation, evaluation of this assumption has been performed. To be able to find out what skin looks like, system has to generate statistical model of skin color. A training set of 54 skin samples (37,020 pixels) and 28 background samples (23,229 pixels) used to generate such models. This paper discusses two types of statistical models - Single Gaussian Model and Gaussian Mixture Model. Performance of both these model has been compared and the best fit model for given dataset has been used. Training dataset was collected using University of Stirling's face database and several images from internet is used. Since dataset comes from different sources, it might result in some unknowns in dataset. One way to eliminate such unknowns is to separate color information from intensity or try to reduce effect of illumination by normalizing color information. To reduce effect of unknowns, this paper uses normalized-rgb (Red, Green, Blue) (illumination is normalized across three color, so effect is reduced), HSV (Hue, Saturation, Value) space (intensity and chromaticity part are independent) and CIE-xyz (Commission Internationale de l'Elcairage) (Machine independent) color spaces. Chrominance models in all three color spaces have been generated and compared to find which color space best suits the selected dataset.
\end{abstract}

\section{Keywords}

Bayes Rules, Face Detection, Golden Ratio, Normalized RGB

\section{INTRODUCTION}

Face recognition has become an important area of research in Computer Vision and Image processing. Any human tracking or identification system first requires location or detection of faces. Project objective is to fulfil this requirement of locating human face. The overall aim of the system is to automatically locate human faces by using skin color classification technique.

This paper also carries out performance evaluation of three chrominance spaces (normalized - RGB, HSV, CIE-XYZ) and determines which of the two statistical model (Single Gaussian Model (SGM) or Gaussian Mixture model (GMM)) best fits the skin distribution space. To fulfil these aims following steps need to be followed:

- Collection of training dataset to train statistical model that represents the skin and background distribution. This training dataset will include skin pixels from two sources face database of University of Stirling [16] and from the images on the internet. Special care has been taken to eliminate effects of flashlights and light conditions.

- Transformation of dataset into selected chrominance space. To have confident solutions from the skin color information and to achieve robustness against flash light effects and lighting conditions, the proposed research uses three color spaces using normalized RGB, HSV, and CIE-xyz.

- Build a statistical model (SGM and GMM) to represent skin and background dataset.

- Separate skin and non-skin region in the give image.

- Identify total number of disjoint skin regions by analysing connectivity of pixels.

- Calculate height and width of all identified disjoint skin region and if the height to width ratio of the given skin regions fall with the given threshold values (calculated by Golden ration), the region can be classified as face.

- Calculate TPR, FPR and draw ROC curves under all threecolor spaces and for both statistical model, to carry out performance evaluation of color spaces and models.

The programming language used to carry out this procedure is Matlab. It has various image processing, data representation and statistical functions; Functions from all these three different areas is unlikely to available together in other programming language, thus it makes Matlab best suitable for the proposed research.

\section{DESIGN APPROACH}

This chapter outlines systematic design procedure during compilation period. It specifies the proposed approach in generation of training data set, color space transformation, generation of statistical model and steps taken by me for skin area recognition and finally detection of face.

\subsection{TRAINING DATASET}

While using feature based skin color detection here the approach is to recognize skin area, it becomes necessary to train skin filter to differentiate skin pixels form the background area. All images in training samples is transformed into JPEG format because it is one of the most commonly used formats [18]. In addition, JPEG file characteristics: color information is stored using $24(3 \times 8)$ bits, that is, 8 bit each for R, G and B [17]. If JPEG file is read in Matlab it returns a three dimensional matrix $[x, y, z]$, i.e. each dimension represent one of the Red, Green and Blue color values of all pixels in an image. It becomes computationally efficient and easy to distinguish individual $R, G$, and $\mathrm{B}$ values from a JPEG file. To generate the proposed dataset 
for skin domain, University of Stirling's database [16] and several images from the internet is used and 54 samples of skin (37020 pixels) from 54 images are also collected. The Fig.1 shows some of the skin samples used as training data set in compilation.

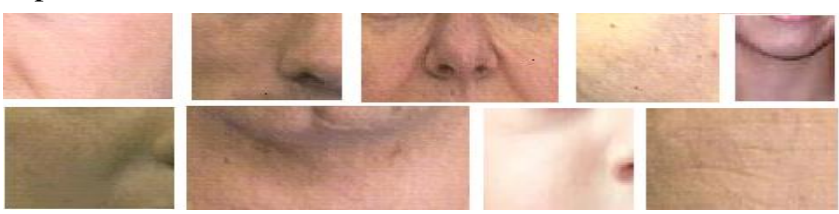

Fig.1. Skin sample images under varying lighting conditions

The background dataset includes samples (small regions) of the background area present in various images in the training set. For the background dataset, paper has collected samples from 28 images (23229 pixels). The Fig. 2 shows some of the background samples.

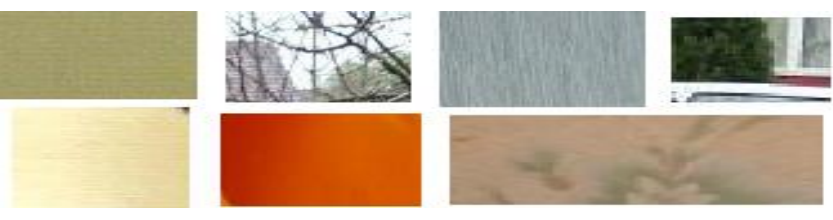

Fig.2. Background sample images

\subsubsection{Color Space Transformation:}

Performance of any statistical model depends on the color space used. In normalized RGB space chromaticity R, G and B are defined as:

$$
\begin{aligned}
& r=\frac{R+G+B}{R} \\
& g=\frac{R+G+B}{G} \\
& b=\frac{R+G+B}{B}
\end{aligned}
$$

where, R, G and B represent the red, green and blue components that describe the pixel color, for the pixel belonging to training data set.

To perform this transformation in compilation, this paper uses $r g b 2 h s v($ ) function of Matlab.

\subsubsection{RGB to CIE xyz Transformation:}

Conversion of RGB to CIE XYZ primaries values can be easily performed using matrix transformation of the following form.

$$
\left[\begin{array}{c}
X \\
Y \\
Z
\end{array}\right]=\left[\begin{array}{lll}
X_{r} & Y_{r} & Z_{r} \\
X_{g} & Y_{g} & Z_{g} \\
X_{b} & Y_{b} & Z_{b}
\end{array}\right] *\left[\begin{array}{c}
R \\
G \\
B
\end{array}\right]
$$

where, $X, Y, Z$ are the described CIE tri-stimulus values, $R, G$, and $B$ represents the red, green and blue color value of a pixel and the $3 \times 3$ matrix is the measured CIE tri-stimulus values for your CRT's three channels (i.e. $X_{r}, Y_{r}, Z_{r}$ are the measured CIE tri-stimulus values for the red channel at maximum emission) [7]. Below are the values used for $3 \times 3$ matrix in compilation, these values are obtained from $[20,21]$.

$$
\left[\begin{array}{ccc}
3.240479 & -1.537150 & -0.498535 \\
-0.969256 & 1.875992 & 0.041556 \\
0.055648 & -0.204043 & 1.057311
\end{array}\right]
$$

Once $X Y Z$ primaries are obtained, the chromaticity of a color was then specified by the two derived parameters $x$ and $y$ which are functions of all three tri-stimulus values $X, Y$ and $Z$. Third coordinate, $z$, can also be defined but is redundant since $x+y+z=1$

$$
\begin{aligned}
& x=\frac{X+Y+Z}{X} \\
& y=\frac{X+Y+Z}{Y} \\
& z=\frac{X+Y+Z}{Z}
\end{aligned}
$$

Using Bayes Rule, skin probability of a pixel is identified. Mathematically, Bayes' rule states [23]

$$
\text { posterior }=\frac{\text { likelihood } \times \text { prior }}{\text { marginal likelihood }}
$$

Or, in symbols,

$$
P(R=r \mid e)=\frac{P(e \mid R=r) P(R=r)}{P(e)}
$$

where, $P(R=r \mid e)$ denotes the probability that random variable $R$ has value $r$ given evidence $e$. The denominator is just a normalizing constant that ensures the posterior adds up to 1 ; it can be computed by summing up the numerator over all possible values of $R$, i.e., $P(e)=P(R=0, e)+P(R=1, e)+\ldots \ldots . .=s u m \_r$ $P(e \mid R=r) P(R=r)$. This is called the marginal likelihood (since we marginalise out over $R$ ), and gives the prior probability of the evidence".

In compilation, once the prior probability density estimates for skin $P(x \mid S)$ and background $P(x \mid B)$ are obtained for test image pixels using Gaussian (SGM and GMM) probability density functions $(p d f)$, the probability of a test image pixel belonging to skin $S$ can be calculated using Bayes rule.

$$
P(S \mid x)=\frac{P(x \mid S) P(S)}{P(x \mid S) P(S)+P(x \mid B) P(B)}
$$

where, $S$ and $B$ denotes classes of skin and background, $P(x \mid S)$ and $P(x \mid B)$ are the prior probabilities of skin and non-skin which are computed using Gaussian probability density functions. $P(S)$ and $P(B)$ are class probabilities which are assumed to be known. Class probability of skin $P(S)$ is set to reflect the size of skin area in the test image and background probability $B$ is set to $1-P(S)$. A test image pixel is set to be classified as belonging to skin area if probability $P(S \mid x)$ is above certain threshold. The Fig.3 shows test images in which skin pixels are identified and are made red. To make skin pixel to appear red, the paper nullifies the green and blue values and made red values to 1 for all those pixels identified as skin.

\subsection{MORPHOLOGICAL OPERATIONS}

The proposed system carries out morphological operation to get rid of unwanted small-scattered area falsely identified as skin region. Some of these falsely selected scattered areas are as shown by green circles in Fig.4. As morphological operation are carried out on binary images. The first step was to generate a binary image such that all the pixels in the test image those are 
identified as skin will have a value 1 , while all other pixels will have a value 0 . The Fig. 4 shows binary image generated with all identified skin pixels are 1's and other pixels are 0 's.

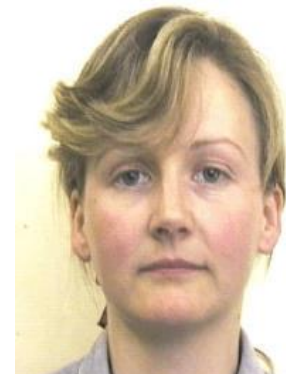

(a)

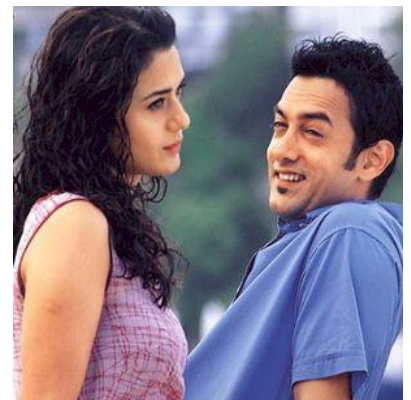

(c)

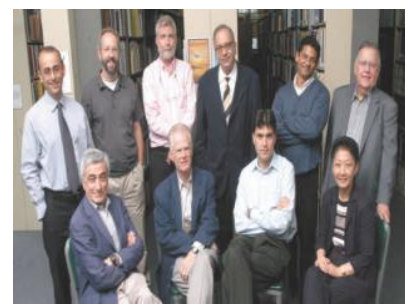

(e)

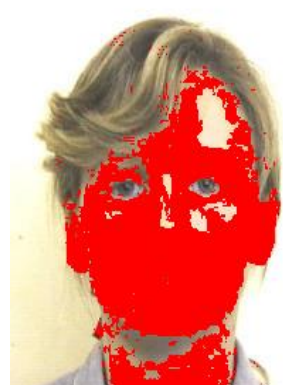

(b)

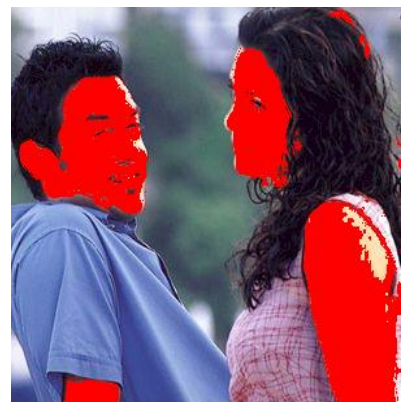

(d)

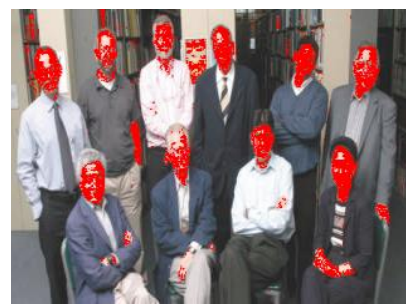

(f)

Fig.3. Image a, c, e are the original images and b, $d, f$ are the images in which identified skin area marked in red color

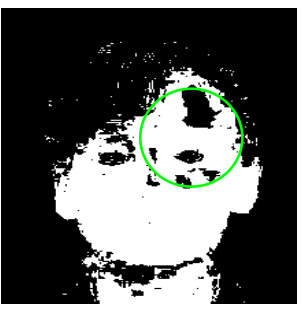

(a)

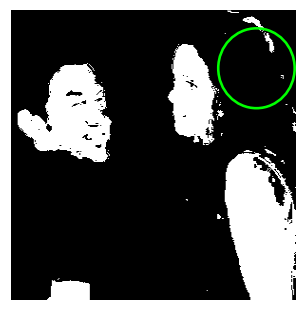

(b)

Fig.4. Binary images generated with all identified skin pixels are 1's and other pixels are 0's. Green circle shows scattered pixels falsely identified as skin region

Now to get rid of unwanted region morphological erosion operation is carried out on the binary image. To carry out this operation, bwmorph() function is used in matlab, this function carries out morphological operation on binary image. Syntax of this function is:

$$
\text { bw2 = bwmorph }(b w 1, \text { operation, } e)
$$

First argument, $b w 1$, is the binary, second argument, operation, specifies the morphological operation to be carried, in our case it was 'erode' and the third argument specifies the

number iterations ( how many times the specified operation is to be carried out on the given image). Erosion operation was carried out two times (no of iterations $=2$ ). By Dilation, we can erode foreground area and increases background area. The Fig. 5 shows dilation operation carried out on images in Fig.4. To achieve this results dilation operation was performed for three times on the images in Fig.4.
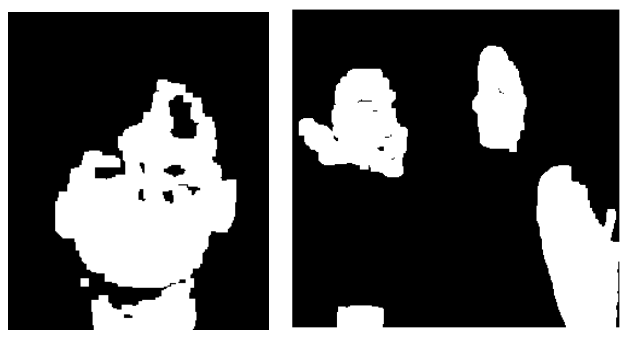

Fig.5. Image after dilation operation. Holes generated between skin regions due to erosion operation are eliminated

\subsection{FACE DETECTION}

Once skin pixels are separated from non-skin pixel, there are number of potential candidates that could be marked as face region. In order to obtain the refined face region, method suggested by [3], [4] is used called a Golden ratio method.

\subsubsection{Connectivity Analysis \& Golden Ratio:}

Using skin segmented image, it can be identified whether the pixels belongs to skin or not, but it is difficult say whether the pixel belongs to Face or not. One cannot say anything about it at pixel level. According to Sandeep et al. [4]

To find the connected regions, one can group all the skin pixels in the image based on an 8-connected neighbourhood. Once we have identified connected regions next step is to classify each of these regions as a human face or not. This could be done by finding height to width or width to height ratio of all the connected region and if the ratio falls within the range of wellknown Golden ratio $=\left(1+(5)^{0.5}\right) / 2 \pm$ some tolerance then the identified region could be considered as a face. Height of the region can be found by following below steps as stated by [4]:

- Find the centre point of the region. This can be found by the average of the coordinate of all the pixels in that region.

- Once centre point is found, for finding height $y$-coordinate of the centre point is subtracted from the $y$-coordinates of all pixels in the region.

- Find the average of all the positive $y$-coordinates and negative $y$-coordinates separately.

- Add the absolute values of both the averages and multiply by 2 . This gives the average height of the region.

- Average width of the region can be found similarly by using $\mathrm{x}$-coordinates. Once the refined face region(s) is identified next step is to draw a rectangle around the identified regions to mark the finding done by the compilation. To draw a rectangle I used a matlab rectangle(), syntax of which is

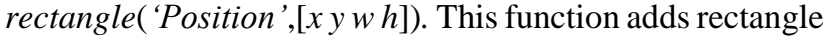
at the specified location. Arguments $x$ and $y$ gives the starting point from were rectangle will be drawn. Argument $w$ and $h$ will specify the width and height of the rectangle. 
The proposed system uses the values for $x, y, w$ and $h$, stated below

$x=$ minimum $(x$-coordinates in the given region)

$y=$ minimum $(y$-coordinates in the given region)

$w=$ width of the region found using above four steps.

$h=$ height of the region found using above four steps.

It is clear from images in Fig.6 that golden ratio works well with any size of faces and for any number of faces in the image. However, there are some false alarms.

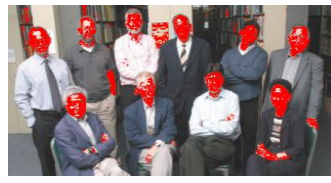

(a)

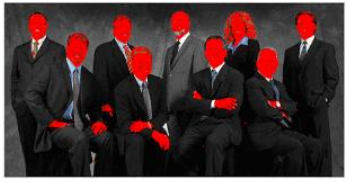

(c)

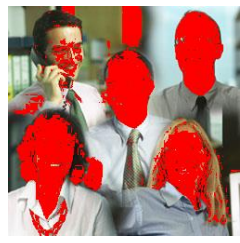

(e)

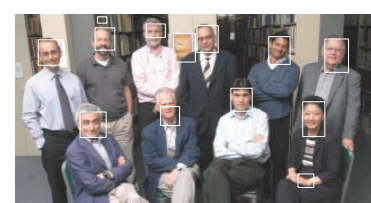

(b)

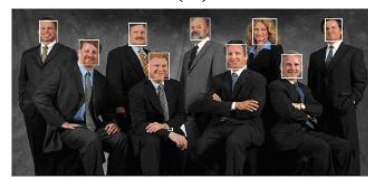

(d)

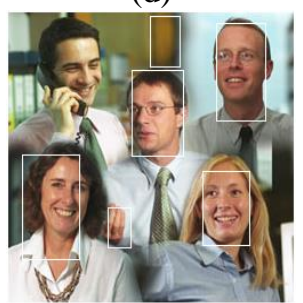

(f)
Fig.6. (a)(c)(e) are images with identified skin region marked with red pixels and $(b)(d)(f)$ are same images with recognised face region marked with rectangles.

\section{TESTING AND RESULTS}

Here the selection of test images and generation of ground truth images, is discussed. The performance of Single Gaussian Model and Multi Gaussian Models is evaluated using testing and training dataset. Once it has been found which chrominance model works well with dataset, this model is used to carry out performance evaluation of three chrominance spaces, namely normalised-RGB, HSV and CIE-XYZ. Finally, the effectiveness of Golden ratio in localising face region is tested.

\subsection{TESTING SET}

In section 2 , the practices adopted by the proposed work towards selection of training dataset is discussed. In this section, the proposed work give reasons for selection of only specific type of images for testing purpose. Skin classification is done using Bayes rule,

$$
P(S \mid x)=\frac{P(x \mid S) P(S)}{P(x \mid S) P(S)+P(x \mid B) P(B)}
$$

where, $P(S)$ and $P(B)$ are the class probabilities of Skin and Background respectively and values for these class probabilities are manually set to reflect the size of skin and non-skin regions in the test image respectively. What if there are any discrepancies in setting values for these Class probabilities? Answer to this question is given next sub-section.

\subsubsection{Effect of Discrepancies in Setting Class Probabilities:}

If the class probabilities are not correctly approximated to reflect the size of skin and non-skin region then FPR (False Positive rate) might increase. FPR (False Positive rate) is the rate of non-skin pixels identified as skin, high FPR results when $P(S)$ (skin class probability) is assigned a value larger than its actual size and since $P(B)=1-P(S)$ values of $P(B)$ will be small than its actual size this might result that a non-skin pixel may be given a higher probability of belonging to skin class. Similarly, TNR (True negative rate) is the rate of skin pixels not correctly identified; this might occur if $P(S)$ is assigned a value small than its actual size. The Fig.7 shows effects, resulting due to discrepancy in assigning class probabilities.

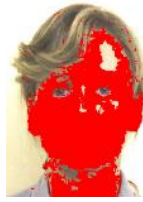

(a)

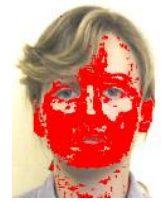

(b)

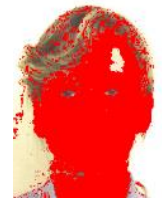

(c)
Fig.7. (a) Image with correctly identified skin pixels, with correct estimation of skin class probability $P(S)=0.5$,

(b) Image with high TNR, occurred because too small estimation of skin class $P(S)=0.2$, (c) Image with high FPR, occurred because too high estimation of skin class $P(S)=0.8$

\subsubsection{Avoiding Class Probabilities Discrepancies and Automation of Process:}

In large and complex images that have multiple skin regions it becomes sometimes difficult to approximate correct amount of skin region, assigning wrong estimation to skin class results in discrepancies as mentioned in above section, also it is very tedious to manually assign value for skin class each and every time for new test Image. To overcome this skin region estimation problem and to automate the entire process, the test images which are not complex, and have equal amount of skin class approximation is considered. Therefore, the test images are selected with only one skin area (face). Size of all faces in test images is same as they are taken from same distance and same angle. For testing, pictures from University of Stirling's database is collected. From this database, images of 10 females and 10 males is selected. Since, training dataset for skin includes only white skin from Europe and Asian origins, so selected 20 test images contains faces with only white skins. All pictures in University of Stirling's database are in GIF format with dimension of $440 \times 550$. The images are transformed into JPEG format and the size of these images is reduced to $130 \times 150$ to minimize computation. The Fig. 8 shows test images.

\subsection{PERFORMANCE ANALYSIS CHROMINANCE MODELS}

In order to say which pixel belongs to skin and which does not, the proposed method manually crops the test images and converted them into binary images; the resulting images shown in Fig.9, which constitutes ground truth images. To compare performance of SGM and GMM using the proposed algorithm, ground truth images are used. 


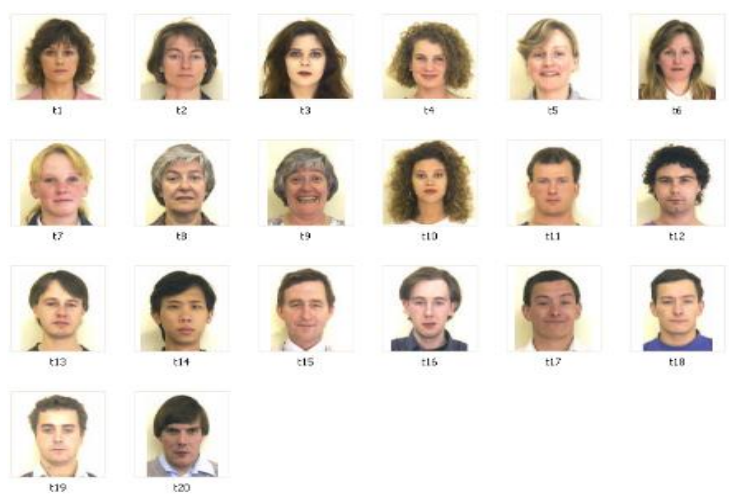

Fig.8. Test image set

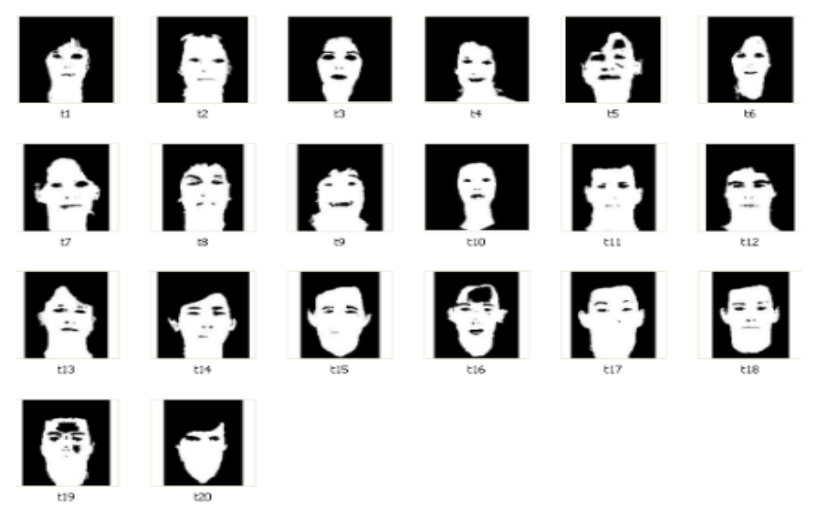

Fig.9. Ground truth images of test images (see, Fig.8)

Once binary skin classification images are obtained through the algorithm, they are compared with ground truth images. The True Positive (TPR) and False Positive (FPR) rates are obtained by,

$$
\begin{gathered}
T P R=\frac{T P}{S} \\
F P R=\frac{F P}{N S}
\end{gathered}
$$

where, $T P$ is number of true positive (pixels correctly assigned to the skin class). FP is the number of false positives (non-skin pixels wrongly assigned to skin). $S$ is the total of skin pixels and $N S$ the totals of non-skin pixels. These rates TPR and FPR are calculated using both models for all test images. Through this computation we get vector of measures $P=(T P R, F P R)$ that express performance of the given model. In this context for comparison of SGM and GMM, ROC curve is suggested by [5], through which we can analyse performance of both the model together. For drawing ROC, TPR and FPR is calculated for all images using different threshold (threshold value is set as per $P(S \mid x)$. Thus, by using $K$ different thresholds, $K$ point vectors $P_{k}$ $=\left(T P R_{k}, F P R_{k}\right)$ are obtained which, when plotted, results in a ROC curve for the specific model with respect to test image. The ROC curves for all test images under both models is calculated.

The Fig.10(a) and Fig.10(b) shows ROC curves for test images 4 and 17 respectively. It is clear form of the ROC curve that both SGM and GMM have almost same TPR. However, FPR in GMM curve (orange dotted line) becomes constant at certain threshold, as shown by green circle. Nevertheless, FPR for SGM (blue line) keeps increasing as threshold valued decreases. FPR reaches almost near to 0.9 . Some thresholds images for images 4 and 17 are shown in Fig. 8 and Fig. 9 shows. Moreover, from the threshold images it is very clear that SGM images have higher FPR rate.

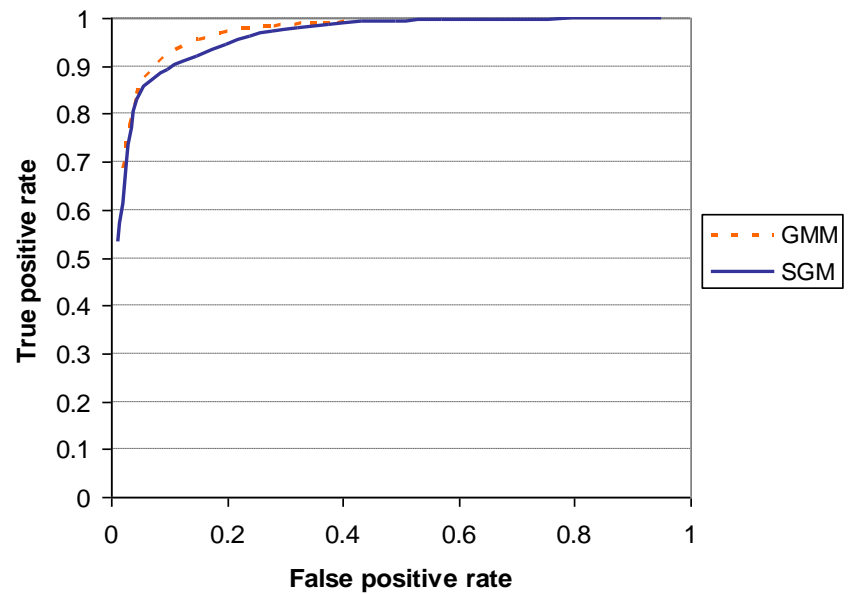

(a)

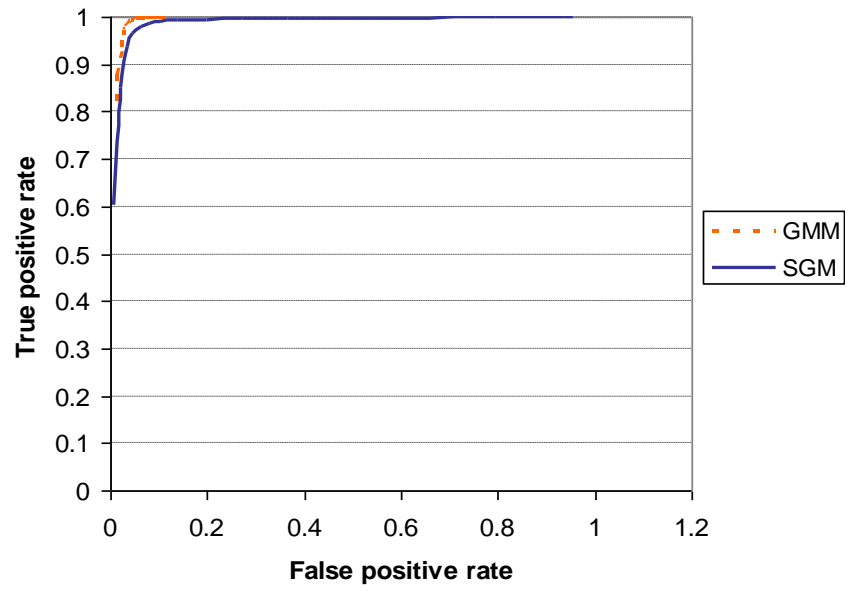

(b)

Fig.10. (a) ROC curve for test image 4 (b) ROC curve for test image 17
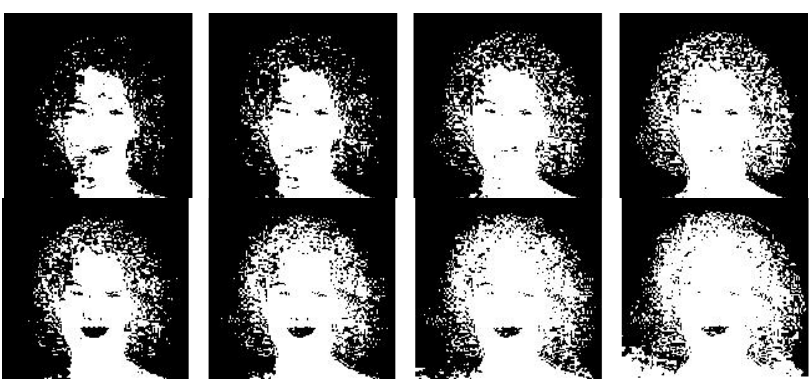

Fig.11. Image 4's threshold images for GMM (1 row) and SGM ( 2 row). Threshold values for each column are $0.7,0.6,0.5$, and 0.4 respectively

Clear results are obtained from the ROC curves of all images. It was evident that SGM had very high FPR and thus they perform badly as compared to GMM and for several images, TPR at a given threshold for SGM was marginal low as compared to GMM. This marginal low TPR will not have any effect on the images with large skin area. Nevertheless for the images with 
small skin area (small faces), this low TPR can have adverse effect.

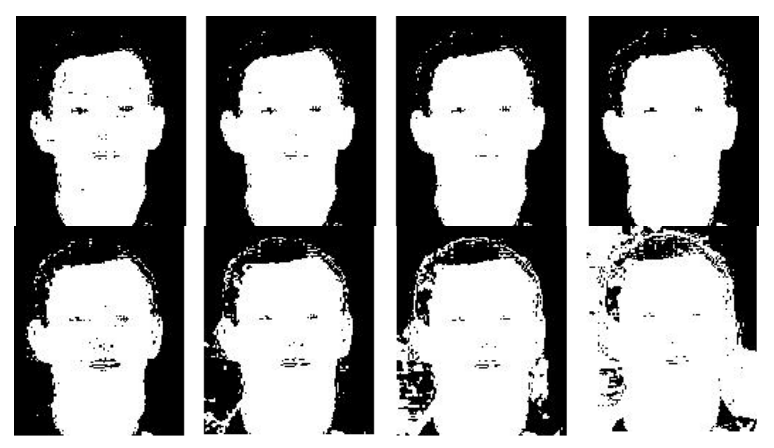

Fig.12. Image 17's threshold images for GMM ( $1^{\text {st }}$ row $)$ and SGM (2 row). Threshold values for each column are $0.7,0.6$, 0.5 , and 0.4 respectively

\subsection{PERFORMANCE ANALYSIS CHROMINANCE SPACES}

OF

In this section, the performance of three chrominance spaces namely is compared against normalised RGB, HSV and CIE XYZ. As discussed in section 3 training datasets and testing images are first transformed into a selected color space and then results are generated for that color space. To generate results for a given color space, ground truth and Gaussian mixture model (because they were identified to perform well for dataset) is used. Once binary skin classified images are obtained by the proposed algorithm in a selected color space using GMM, they are compared with the ground truth images. The FPR and TPR are calculated for each images in the test set. This results into vector $P(T P R, F P R)$ that would express the performance of given color space for training set. Vector $P(T P R, F P R)$ is calculated for all three color spaces and they were compared. The Table. 1 shows calculated TPR and FPR for all three-color spaces. For this calculation, fixed threshold value of 0.55 is used, because it is middle range value where we can find highest TPR and low FPR.

Table.1. Calculated TPR and FPR rates for all three color spaces using GMM

\begin{tabular}{|c|c|c|c|c|c|}
\hline \multicolumn{2}{|c|}{ SV } & \multicolumn{2}{c|}{ Normalized - RGB } & \multicolumn{2}{c|}{ CIE-XYZ } \\
\hline TPR & FPR & TPR & FPR & TPR & FPR \\
\hline 0.9228 & 0.2374 & 0.8518 & 0.2654 & 0.7931 & 0.2256 \\
\hline 0.9516 & 0.0654 & 0.9699 & 0.0832 & 0.9638 & 0.0837 \\
\hline 0.8128 & 0.3118 & 0.9151 & 0.3241 & 0.9063 & 0.2794 \\
\hline 0.9726 & 0.1656 & 0.9685 & 0.1939 & 0.9454 & 0.1486 \\
\hline 0.9009 & 0.0921 & 0.9357 & 0.1135 & 0.9388 & 0.1065 \\
\hline 0.9895 & 0.1777 & 0.994 & 0.2053 & 0.992 & 0.1649 \\
\hline 0.989 & 0.1263 & 0.9852 & 0.1273 & 0.9745 & 0.0949 \\
\hline 0.9644 & 0.0878 & 0.9728 & 0.0995 & 0.9637 & 0.1042 \\
\hline 0.9662 & 0.0579 & 0.9699 & 0.0646 & 0.9647 & 0.0705 \\
\hline 0.9793 & 0.2022 & 0.7551 & 0.23 & 0.7528 & 0.1755 \\
\hline 0.9938 & 0.0639 & 0.9395 & 0.0785 & 0.9359 & 0.076 \\
\hline 0.9857 & 0.0628 & 0.9906 & 0.0643 & 0.9874 & 0.0693 \\
\hline 0.9001 & 0.0627 & 0.9335 & 0.0708 & 0.8903 & 0.0694 \\
\hline
\end{tabular}

\begin{tabular}{|c|c|c|c|c|c|}
\hline 0.9714 & 0.0445 & 0.8289 & 0.0501 & 0.8141 & 0.0494 \\
\hline 0.9936 & 0.0897 & 0.9985 & 0.105 & 0.9952 & 0.1078 \\
\hline 0.9578 & 0.0753 & 0.9596 & 0.0703 & 0.9691 & 0.0894 \\
\hline 0.9911 & 0.0363 & 0.9925 & 0.0409 & 0.9893 & 0.0429 \\
\hline 0.9888 & 0.0438 & 0.9948 & 0.0462 & 0.9894 & 0.0495 \\
\hline 0.7673 & 0.0446 & 0.8534 & 0.0525 & 0.8515 & 0.0574 \\
\hline 0.9329 & 0.0243 & 0.9448 & 0.0267 & 0.9451 & 0.0283 \\
\hline
\end{tabular}

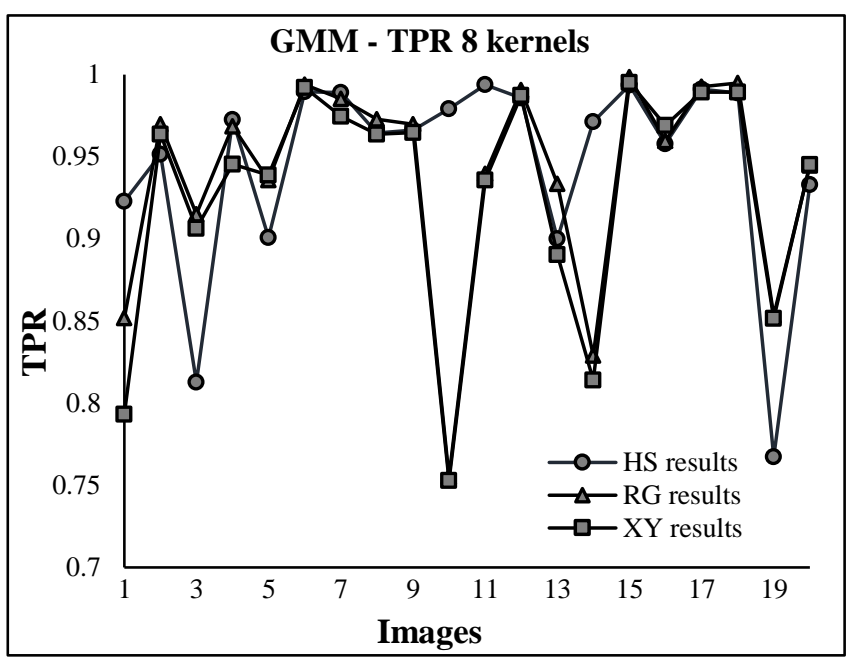

(a)

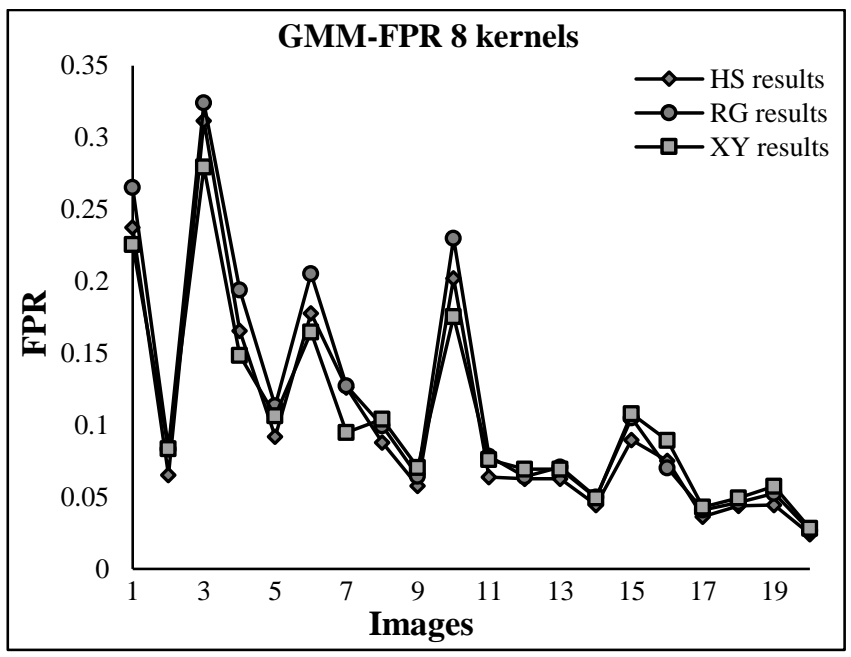

(b)

Fig.13. (a) TPR calculated for test set under three color spaces (b) FPR calculated for test set under three color spaces

From Fig.13 and Table. 1 it is rather difficult to say which color space has better performance. Because all three color, space seems to have almost equal results. It is believed that the difference between three-color spaces using GMM and the proposed dataset is very subtle. The reason is GMM very well defines the skin and non-skin distribution over all three-color spaces. Therefore, to compare performance of three-color spaces the proposed system uses SGM, which has results in high FPR. So the model, which yields list FPR under SGM, will be considered to perform best for selected dataset. The Table. 2 shows calculated TPR and FPR rates using SGM for three color spaces. 
Table.2. Calculated TPR and FPR rates for all three-color spaces using SGM

\begin{tabular}{|c|c|c|c|c|c|}
\hline \multicolumn{2}{|c|}{ HSV } & \multicolumn{2}{c|}{ Normalised RGB } & \multicolumn{2}{c|}{ CIE-XYZ } \\
\hline TPR & FPR & TPR & FPR & TPR & FPR \\
\hline 0.5689 & 0.2395 & 0.8442 & 0.1341 & 0.9156 & 0.207 \\
\hline 0.8947 & 0.0995 & 0.9371 & 0.0596 & 0.9641 & 0.1183 \\
\hline 0.7538 & 0.2632 & 0.8655 & 0.1985 & 0.9708 & 0.2683 \\
\hline 0.8568 & 0.2706 & 0.8915 & 0.0969 & 0.9198 & 0.1581 \\
\hline 0.6378 & 0.161 & 0.9019 & 0.0809 & 0.9492 & 0.1263 \\
\hline 0.9337 & 0.2527 & 0.8587 & 0.1117 & 0.9217 & 0.1669 \\
\hline 0.6664 & 0.1598 & 0.8383 & 0.0628 & 0.8966 & 0.0813 \\
\hline 0.8742 & 0.0529 & 0.9247 & 0.0822 & 0.9544 & 0.1352 \\
\hline 0.9083 & 0.0341 & 0.8353 & 0.0548 & 0.8943 & 0.109 \\
\hline 0.512 & 0.297 & 0.7843 & 0.1163 & 0.8836 & 0.1787 \\
\hline 0.7496 & 0.0788 & 0.9172 & 0.0607 & 0.9527 & 0.1021 \\
\hline 0.7915 & 0.0395 & 0.969 & 0.0506 & 0.9834 & 0.0757 \\
\hline 0.8437 & 0.0789 & 0.8005 & 0.0535 & 0.8713 & 0.0806 \\
\hline 0.65 & 0.0373 & 0.7279 & 0.0401 & 0.8249 & 0.0594 \\
\hline 0.8503 & 0.0931 & 0.9284 & 0.0864 & 0.9606 & 0.1403 \\
\hline 0.2353 & 0.0254 & 0.7993 & 0.0483 & 0.9435 & 0.1242 \\
\hline 0.9598 & 0.0362 & 0.9364 & 0.0349 & 0.9641 & 0.054 \\
\hline 0.8857 & 0.0255 & 0.9501 & 0.0383 & 0.9753 & 0.0556 \\
\hline 0.5688 & 0.0254 & 0.8118 & 0.0471 & 0.9114 & 0.0984 \\
\hline 0.7346 & 0.0315 & 0.8503 & 0.0214 & 0.9173 & 0.0409 \\
\hline
\end{tabular}

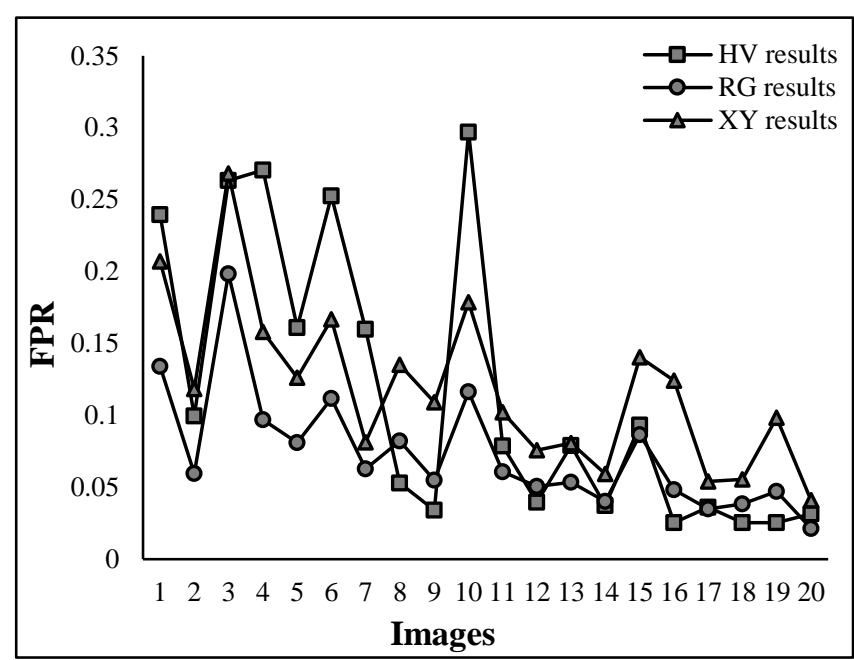

Fig.13. FPR calculated for test set images under all three-color space using SGM

From, Table. 2 and Fig.13 (Plot of FPR under all three-color spaces), It is conclude that normalized-RGB had the least FPR rate among the 20 images and thus would perform best, followed by HSV. CIE-XYZ had the Highest FPR rate for about 12 images out of 20 and thus would perform worst compared to other two models for dataset.

\subsection{EFFECTIVENESS OF GOLDEN RATIO}

To check effectiveness of Golden ratio in face recognition, the research uses 10 test images (shown in Fig.14), which in all has 61 faces in them. Test images include faces of different sizes and oriented at different angles. All this test images are taken from internet. All images are having different backgrounds. The test experiment calculate how many faces are correctly identified and also will calculate amount of false alarms. Out of 61 faces, 12 faces were not detected through the use of Golden ratio. This means face detection rate was almost $80 \%$ for given test images.

There were 13 false alarms in 10 images, which mean 1.3 per image. The research concluded that using Golden ratio it is not possible to separate closely located skin regions. As seen in below images Fig.14(d) and Fig.14(i), were skin region of hand and face are intersecting each other. In such cases using Golden ration own its own will not be effective; we would need combination of some other geometric methods (edge detection) and golden ratio. Also using golden ratio, it becomes difficult to recognize side faces as seen in below image Fig.14(a) and Fig.14(b).

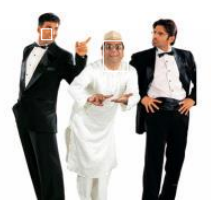

(a)

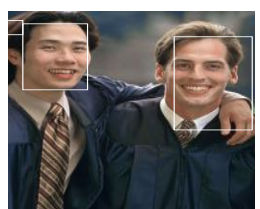

(d)

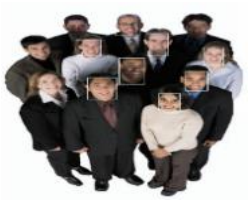

(g)

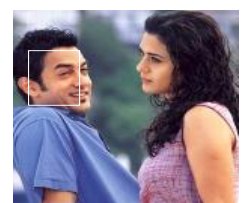

(b)

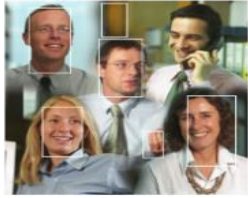

(e)

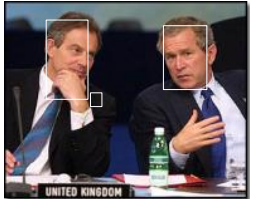

(h)

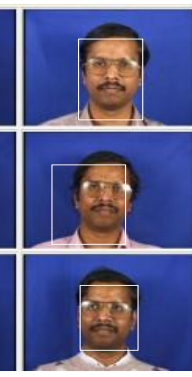

(j) (c)

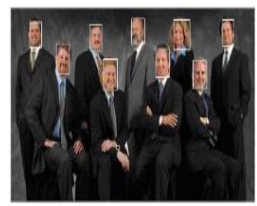

(f)

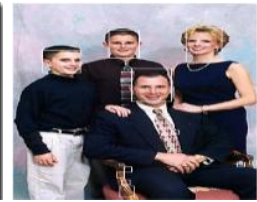

(i)

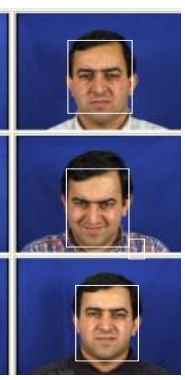

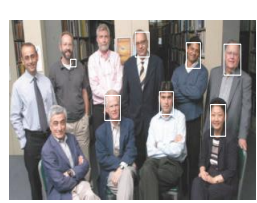
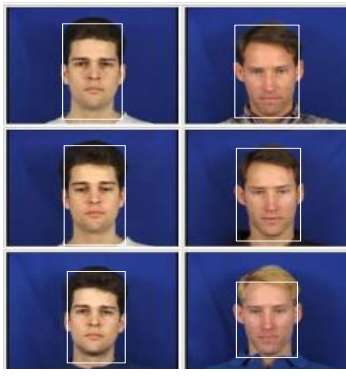

Fig.14. Test Images with recognized face region marked with white rectangle. This test images have in all 61 faces, out of which 49 faces are correctly located

In 61 faces, 12 faces were not identified and 13 false alarms were raised in 10 images. Thus the performance of Golden ratio was quite encouraging. 


\section{CONCLUSION}

The initial goal of the project was to develop an algorithm that would detect all faces in the given colored image. Through the implementation, the proposed system manages to achieve this goal only to a certain extent, but the research and implementation are on the right track and have gone far. Through the implementation, the proposed system managed to show that GMM has lower false positive rates as compared to SGM. For the given datasets, it was identified that normalised RGB space best describes the skin color with least overlap between skin and non-skin color distribution. Comparing three color spaces (normalised-RGB, HSV and CIE-XYZ); it was found that normalised-RGB had least FPR, followed by HSV and CIE-xyz had the highest FPR. The results are encouraging with Golden ratio rule for face detection. For 61 faces, the success rate of $80 \%$ with 1.3 false alarms per image is achieved. This report describes various important techniques relevant to face detection and list how some of this techniques are implemented. After implementation, it also compares performance of some of these techniques.

\section{FUTURE WORK}

The skin dataset is very small, thus making the proposed algorithm inefficient for images with skin intensity that does not fall within range of skin dataset. The proposed system includes only the white skin samples in the dataset, which brings another constraint to the proposed algorithm, it will not work with all ethnic groups. Unfortunately, due to unavailability of different face databases to me, the proposed system is not able to include large skin samples in dataset. Further the work is intended to test the algorithm with large training dataset covering skin from large ethnic groups.

In Bayes Rule we have to manually specify value for class probability of skin. This manual process needs to be made automated. For this, a new functionality in the proposed algorithm is added. Using Golden ratio own its own is not efficient to detect face regions. As identified, it works only for disjoint skin regions and fails if two skin regions are joint, e.g. if two faces in an image touch each other, algorithm will fail to identify both the faces. For this, other features of face such as eyes or nose are used in accordance with Golden ratio rule for efficient detection of face.

\section{ACKNOWLEDGMENT}

I would like to thank Mr. Geoff Dowlling (University of Westminster, Harrow, UK) for supervising my project. His help has enabled me to transform his suggestions into implementations.

\section{REFERENCES}

[1] Erik Hjelmas and Boon Kee Low, "Face Detection: A Survey", Computer Vision and Image Understanding, Vol. 83, No. 3, pp. 236-274, 2001.

[2] Stan Z. Li and Anil K. Jain, "Handbook of Face Recognition", $2^{\text {nd }}$ Edition, Springer, 2011.
[3] V. Govindaraju, "Locating Human Faces in Photographs", International Journal of Computer Vision, Vol. 19, No. 2, pp. 129-146, 1996.

[4] K. Sandeep and A.N. Rajagopalan. "Human Face Detection in Cluttered Color Images using Skin Color and Edge Information", Available https://www.cse.iitb.ac.in/ sharat/icvgip.org/icvgip2002/p roceedings/166.pdf.

[5] Tiberio S. Caetano, Silvia D. Olabarriaga and Dante A.C. Barone, "Performance Evaluation of Single and MultipleGaussian Models for Skin Color Modelling", Proceedings of 15th Brazilian Symposium on Computer Graphics and Image Processing, pp. 112-117, 2002.

[6] Jean-Christophe Terrillon, Mahdad N. Shirazi, Hideo Fukamachi and Shigeru Akamatsu. "Comparative Performance of Different Skin Chrominance Models and Chrominance Spaces for the Automatic Detection of Human Faces in Color Images", Proceedings of $4^{\text {th }}$ IEEE International Conference on Automatic Face and Gesture Recognition, pp. 105-109, 2000.

[7] Adrian Ford and Alan Roberts, "Color Space Conversions", Available at: https://poynton.ca/PDFs/coloureq.pdf

[8] J.C. Terrillon, Y. Niwa and K. Yamamoto, "On the Selection of an Efficient Chrominance Space for Skin Color-based Image Segmentation with an Application to Face Detection", Proceedings of International Conference on Quality Control by Artificial Vision, Vol. 1, pp. 354-361, 2001.

[9] Alberto Albiol, Luis Torres and Edward J. Delp, "An Unsupervised Color Image Segmentation Algorithm for Face Detection Applications", Proceedings of International Conference on Image Processing, pp. 23-27, 2001.

[10] Sanjay Kr. Singh, D. S. Chauhan, Mayank Vatsa and Richa Singh; "A Robust Skin Color based Face Detection Algorithm"; Tamkang Journal of Science and Engineering, Vol. 6, No. 4, pp. 227-234, 2003.

[11] Sangoh Jeong, "Histogram-Based Color Image Retrieval", Available at: https://ece.uwaterloo.ca/ nnikvand/Coderep/ColorHist/His togram-Based\%20Color\%20Image\%20Retrieval.pdf

[12] Michael Walter, "Extreme Value Theory for Visual Recognition", Available at: http://cs.uccs.edu/ jkalita/work/reu/REU2014/FinalPapers /Moore.pdf

[13] HSV Color Space, Available at: https://en.wikipedia.org/wiki/HSL_and_HSV.

[14] CIE1931 Color Space, Available at: http://en.wikipedia.org/wiki/CIE_1931_color_space.

[15] Psychological Image Collection at Stirling, Available at: http://pics.psych.stir.ac.uk/cgibin/PICS/New/pics.cgi?filename=shopwuwwEh\&shop=clear

[16] JPEG Files, Available at: http://www.csit.fsu.edu/ burkardt/data/jpg/jpg.html

[17] JPEG Format, Available at: http://en.wikipedia.org/wiki/JPEG

[18] Ruye Wang, "The Color Space", Available at: http://fourier.eng.hmc.edu/e180/handouts/color1/node27.h tml, Accessed on 2002. 
[19] Pascal Getreuer, "Color Space", Available at: http://www.mathworks.com/matlabcentral/files/7744/cont ent/colorspace/doc/colorspace.html.

[20] Color Conversion Algorithms, Available at: http://www.cs.rit.edu/ ncs/color/t_convert.html

[21] Ian. T. Nabney. "Netlab Algorithms for Pattern Recognition", $1^{\text {st }}$ Edition, Springer, 2002.

[22] Kevin Murphy, "A Brief Introduction to Bayes Rule", Available at: http://www.cs.ubc.ca/ murphyk/Bayes/bayesrule.html.

[23] Alex Pentland and Tanzeem Choudhury, "Personalizing Smart Environments: Face Recognition for Human Interaction", Available at: http://vismod.media.mit.edu/tech-reports/TR516/ieee_computer.html.

[24] Yi Chan, Richard Harvey and Dan Smith, "Building Systems to Block Pornography", Available at: http://www.bcs.org/upload/pdf/ewic_im99_paper8.pdf.
[25] Paul Bourke, "RGB color space", Available at: http://local.wasp.uwa.edu.au/ pbourke/color/colorspace/, Accessed on 1995.

[26] Demir Gokalp, "Learning Skin pixels in Color Images using Gaussian Mixture", Available at: http://www.cs.bilkent.edu.tr/ guvenir/courses/CS550/Wor kshop/Demir_Gokalp.pdf. .

[27] Ming-Hsuan Yang and Narendra Ahuja, "Gaussian Mixture Model for Human Skin Color and its Applications in Image and Video Database", Available at: https://pdfs.semanticscholar.org/ee04/945d25f21fc1a7cc4 57610a6aff0dee34420.pdf.

[28] Kardi Technomo, "Numerical Example of K-Means", Available at: http://people.revoledu.com/kardi/tutorial/kMean/Numerica lExample.htm. 